Vietnam Journal of Mechanics, VAST, Vol.39, No. 1 (2017), pp. 51-68

DOI:10.15625/0866-7136/7817

\title{
ISOGEOMETRIC ANALYSIS OF LINEAR ISOTROPIC AND KINEMATIC HARDENING ELASTOPLASTICITY
}

\author{
Khuong D. Nguyen ${ }^{1}$, Minh N. Nguyen ${ }^{1}$, Hoa V. Cong ${ }^{1}$, H. Nguyen-Xuan ${ }^{2, *}$ \\ ${ }^{1}$ Ho Chi Minh City University of Technology, VNU-HCMC, Vietnam \\ ${ }^{2}$ Ho Chi Minh City University of Technology (HUTECH), Vietnam \\ *E-mail: ngx.hung@hutech.edu.vn \\ Received February 25, 2016
}

\begin{abstract}
Material nonlinearity is of great importance in many engineering problems. In this paper, we exploit NURBS-based isogeometric approach in solving materially nonlinear problems, i.e. elastoplastic problems. The von Mises model with linear isotropic hardening and kinematic hardening is presented, and furthermore the method can also be applied to other elastoplastic models without any loss of generality. The NURBS basis functions allow us to describe exactly the curved geometry of underlying problems and control efficiently the accuracy of approximation solution. Once the discretized system of non-linear equilibrium equation is obtained, the Newton-Raphson iterative scheme is used. Several numerical examples are tested. The accuracy and reliability of the proposed method are verified by comparing with results from ANSYS Workbench software.
\end{abstract}

Keywords: Isogeometric analysis, NURBS, Rate-independent plasticity, von Mises yield criterion, isotropic hardening, kinematic hardening.

\section{INTRODUCTION}

Metal forming and pre-stressed concrete are examples of plasticity being applied in industry. Plastic deformation is found in a wide variety of materials, such as metals, soils, rocks, martensitic materials and cellular materials, etc. Thus, investigation of plasticity is of great importance in engineering. One key factor in theory of plasticity is the yield criterion, which determines whether a material points is under plastic state or not. There are various yield criteria corresponding to many types of materials, such as von Mises model and Tresca model for ductile metals, Drucker-Prager and Mohr-Coulomb for geomaterials, Cam-Clay model for soils, etc. [1].

The accuracy of nonlinear elasto-plastic analysis strongly depends on the integration scheme. Because the material behaviour is described with a rate-form constitutive equation, an increment solution strategy is often used. In a small strain problem, the

(C) 2017 Vietnam Academy of Science and Technology 
strain rate remains constant during the increment. Krieg and Krieg [2] showed an analytical solution for perfectly plastic von Mises model by using the constant strain rate assumption. Wei [3] derived the stress update formula with the consistent linearization. Szabó [4], who developed the partial analytical solution proposed by Ristinmaa and Tryding [5], achieved a semi-analytical solution for the purely linear isotropic hardening von Mises elastoplastic model.

Elastoplastic problem has been intensively investigated with the aid of numerical methods such as finite element method [6,7], meshfree method [8-10], boundary element method [11,12]. The implicit solution schemes were investigated. This method was proposed by Simo and Taylor [13] in the finite element method context. It exploits the quadratic rate of convergence in the Newton-Raphson iterative process. In spite of the great success on existing numerical methods for the elastoplastic problems, there is still a growing interest in the development of new advanced methods.

In recent years, the isogeometric analysis (IGA) [14,15] has gained much attention from research community as an alternative numerical method to the well-known finite element method (FEM), due to utilizing its exact geometrical modelling and higher order continuity. IGA has been successfully implemented in many applications, such as structural vibrations [16], plates $[17,18]$, shells $[19,20]$, incompressibility [21], electromagnetics [22] and phase fields [23,24], etc. However, to the best knowledge of the authors, the application of IGA in solving the elastoplastic problems has not been much mentioned in literatures. In this paper, our investigation is focused on the elasto-plastic von Mises model with isotropic hardening, and the extension of the present approach to other yield criterion is completely straightforward.

This paper is structured as follows. Section 2 states a brief review of NURBS basis functions. The algorithms for von Mises plasticity model with linear isotropic hardening and kinematic hardening using NURBS-based IGA are presented in Section 3. Several numerical examples are investigated in Section 4. Finally, some concluding remarks are closed in Section 5.

\section{NURBS BASIS FUNCTIONS}

In this section, a brief summary of some technical features of non-uniform rational B-spline (NURBS) is presented. A more detailed description can be found in [25]. A NURBS curve, $\mathbf{C}(\xi)$, of order $p$ is the linear combination of the NURBS basis functions, in which the coefficients are a given set of control points

$$
\mathbf{C}(\xi)=\sum_{i=1}^{n} R_{i, p}(\xi) \mathbf{P}_{i},
$$

where $R_{i, p}(\xi)$ is the univariate NURBS basis functions determined by

$$
R_{i, p}(\xi)=\frac{N_{i, p}(\xi) w_{i}}{\sum_{i=1}^{n} N_{i, p}(\xi) w_{i}}
$$


$\mathbf{P}_{i}=\left(x_{i}, y_{i}\right), i=1,2, \ldots, n$ is the set of $n$ control points and $w_{i}$ is the non-negative weight assigned for the $i^{\text {th }}$ control point, and $N_{i, p}(\xi)$ are the B-spline basis functions of order $p$. Corresponding to the set of $n$ B-spline basis functions of order $p$, a knot vector $\Xi$ with non-decreasing sequence of real numbers in a parametric space, $\xi \in[0,1]$, is defined as

$$
\Xi=\left\{\xi_{1}, \xi_{2}, \ldots, \xi_{n+p+1}\right\} ; \quad \xi_{i} \leq \xi_{i+1} ; \quad i=1,2, \ldots, n+p .
$$

A knot vector is said to be open if the knots are repeated $p+1$ times at the start and end of the vector. For analysis purposes, the open knot vectors are generally used to take advantage of the Kronecker-delta property at the boundary points. Given a knot vector, the univariate B-spline basis function, $N_{i, p}(\xi)$, can be constructed recursively by the Cox-de Boor formulation

$$
N_{i, 0}(\xi)= \begin{cases}1, & \text { if } \xi_{i} \leq \xi<\xi_{i+1} \\ 0, & \text { if } \xi<\xi_{i} \text { or } \xi \geq \xi_{i+1}\end{cases}
$$

and with $p=1,2,3, \ldots$

$$
N_{i, p}(\xi)=\frac{\xi-\xi_{i}}{\xi_{i+p}-\xi_{i}} N_{i, p-1}(\xi)+\frac{\xi_{i+p+1}-\xi}{\xi_{i+p+1}-\xi_{i+1}} N_{i+1, p-1}(\xi) .
$$

The B-spline basis functions constructed from an open knot vector have the interpolation property at both ends of the parametric space. A set of cubic B-spline basis functions constructed from an open knot vector is depicted in Fig. 1.

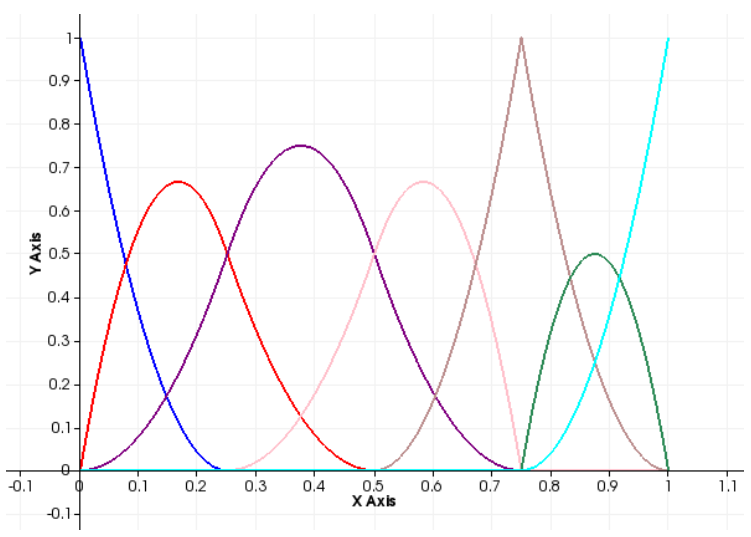

Fig. 1. Cubic basis functions for the open knot vector $\boldsymbol{\Xi}=\{0,0,0,0,0.25,0.5,0.75,1,1,1,1\}$

A NURBS surface, $\mathbf{S}(\xi, \eta)$, of order $p$ in $\xi$-direction and order $q$ in $\eta$-direction can be expressed generally as

$$
\mathbf{S}_{\xi, \eta}=\sum_{i=1}^{n} \sum_{j=1}^{m} R_{i, j}^{p, q}(\xi, \eta) \mathbf{P}_{i, j},
$$

where $R_{i, j}^{p, q}(\xi, \eta)$ stands for the bivariate NURBS basis functions determined from the univariate B-spline basis functions $N_{i, p}$ and $M_{j, q}$ defined on the $\boldsymbol{\Xi}$ and $\mathbf{H}$ knot vectors 
corresponding to $\xi$-direction and $\eta$-direction, respectively

$$
R_{i, j}^{p, q}(\xi, \eta)=\frac{N_{i, p}(\xi) M_{j, q}(\eta) w_{i, j}}{\sum_{i=1}^{n} \sum_{j=1}^{m} N_{i, p}(\xi) M_{j, q}(\eta) w_{i, j}}
$$

and $\mathbf{P}_{i, j}$ are the list of $n \times m$ control points, and $w_{i, j}$ are the assigned weights. The firstorder derivative of $R_{i, p}^{p, q}(\xi, \eta)$, with respect to each parametric variable, e.g. $\xi$, is derived directly from Eq. (7)

$$
\frac{\partial R_{i, j}^{p, q}(\xi, \eta)}{\partial \xi}=\frac{\frac{\partial N_{i, p}(\xi)}{\partial \xi} M_{j, q}(\eta) w_{i, j} W(\xi, \eta)-\frac{\partial W(\xi, \eta)}{\partial \xi} N_{i, p}(\xi) M_{j, q}(\eta) w_{i, j}}{W(\xi, \eta)},
$$

with

$$
\begin{aligned}
& W(\xi, \eta)=\sum_{i=1}^{n} \sum_{j=1}^{m} N_{i, p}(\xi) M_{j, q}(\eta) w_{i, j}, \\
& \frac{\partial W(\xi, \eta)}{\partial \xi}=\sum_{i=1}^{n} \sum_{j=1}^{m} \frac{\partial N_{i, p}(\xi)}{\partial \xi} M_{j, q}(\eta) w_{i, j} .
\end{aligned}
$$

Any higher-order derivatives of the NURBS basis can also be obtained in a similar fashion. The idea of tensor product in Eq. (6) and Eq. (7) is also applicable to build the NURBS solids

$$
\mathbf{S}(\xi, \eta, \zeta)=\sum_{i=1}^{n} \sum_{j=1}^{m} \sum_{k=1}^{l} R_{i, j, k}^{p, q, r}(\xi, \eta, \zeta) \mathbf{P}_{i, j, k},
$$

where the trivariate NURBS basis function is given by

$$
R_{i, j, k}^{p, q, r}(\xi, \eta, \zeta)=\frac{N_{i, p}(\xi) M_{j, q}(\eta) L_{k, r}(\zeta) w_{i, j, k}}{\sum_{i=1}^{n} \sum_{j=1}^{m} \sum_{k=1}^{l} N_{i, p}(\xi) M_{j, q}(\eta) L_{k, r}(\zeta) w_{i, j, k}}
$$

\section{RETURN-MAPPING ALGORITHM FOR VON MISES YIELD CRITERION}

In this section, the Return-mapping algorithm for the von-Mises yield criterion, widely used for metal plasticity, with isotropic hardening is adopted. The total hardening is assumed to be a combination of the isotropic hardening, $H_{i}(\alpha)$, accounting for the dilation of the yield surface, and the kinematic hardening, $H_{k}(\alpha)$, accounting for the translation of the yield surface. We write [6]

$$
\begin{aligned}
& H_{i}(\alpha)=\sigma_{Y}+\theta \xi(\alpha), \\
& H_{k}^{\prime}(\alpha)=(1-\theta) \xi^{\prime}(\alpha), \theta \in[0,1]
\end{aligned}
$$

in which $\sigma_{Y}$ is the initial yield stress in uniaxial tensile test and $\alpha$ is equivalent plastic strain and $\xi(\alpha)$ is the hardening function. The von-Mises yield criterion with combined 
isotropic/kinematic hardening is given by

$$
f(\mathbf{s}, \mathbf{q}, \alpha)=\|\mathbf{s}-\mathbf{q}\|-\sqrt{\frac{2}{3}} H_{i}(\alpha),
$$

where $\mathbf{s}$ is the deviatoric stress, $\mathbf{q}$ defines the center of the von Mises yield surface in deviatoric space.

The evolution law gives

$$
\begin{aligned}
& \dot{\varepsilon}^{p}=\dot{\gamma} \frac{\partial f}{\partial \sigma}, \\
& \dot{\alpha}=-\dot{\gamma} \frac{\partial f}{\partial \xi}, \\
& \dot{\mathbf{q}}=\frac{2}{3} H_{k}^{\prime}(\alpha) \dot{\varepsilon}^{p},
\end{aligned}
$$

in which $\beta=\mathbf{s}-\mathbf{q}$ is the relative stress. Applying the backward Euler time integration, the above rate form can be rewritten in an incremental from as

$$
\begin{aligned}
& \Delta \varepsilon^{p}=\Delta \gamma \frac{\boldsymbol{\beta}}{\|\boldsymbol{\beta}\|}, \\
& \Delta \alpha=\Delta \gamma \sqrt{\frac{2}{3}}, \\
& \Delta \mathbf{q}=\frac{2}{3} H_{k}^{\prime}(\alpha) \Delta \gamma \frac{\boldsymbol{\beta}}{\|\boldsymbol{\beta}\|} .
\end{aligned}
$$

With the notice on Eq. (20), Eq. (21) can be rewritten as

$$
\Delta \mathbf{q}=\sqrt{\frac{2}{3}} H_{k}^{\prime}(\alpha) \Delta \alpha \frac{\boldsymbol{\beta}}{\|\boldsymbol{\beta}\|}=\sqrt{\frac{2}{3}} \Delta H_{k}(\alpha) \frac{\boldsymbol{\beta}}{\|\boldsymbol{\beta}\|} .
$$

Assume that step $n+1$ is plastic, the updated stress is expressed by [6]

$$
\boldsymbol{\sigma}_{n+1}=\frac{I_{1, n+1}}{3} \mathbf{I}+\mathbf{s}_{n+1}=\boldsymbol{\sigma}_{n+1}^{t r}-\Delta \gamma \mathbb{C}: \mathbf{n}_{n+1},
$$

where $\sigma_{n+1}^{t r}$ is the trial stress, which is assumed to be in elastic state as

$$
\boldsymbol{\sigma}_{n+1}^{t r}=\frac{I_{1, n+1}^{t r}}{3} \mathbf{I}+\mathbf{s}_{n+1}^{t r}
$$

and $\mathbf{I}$ is the second order unit tensor, $\mathbf{n}_{n+1}=\frac{\partial f_{n+1}}{\partial \boldsymbol{\sigma}_{n+1}}=\frac{\boldsymbol{\beta}_{n+1}}{\left\|\boldsymbol{\beta}_{n+1}\right\|}$ and $\mathbb{C}$ is the tensor of elastic property

$$
\mathbf{C}=\kappa \mathbf{I} \otimes \mathbf{I}+2 G \mathbf{I}^{d e v},
$$

in which $\kappa$ is the bulk modulus, $G$ is the shear modulus and $\mathbf{I}^{d e v}$ is the deviatoric operator. Due to the pure deviatoric nature of von Mises plastic flow, i.e. $I_{1, n+1}=I_{1, n+1}^{t r}$ and the orthogonality between the hydrostatic and deviatoric plane, one obtains

$$
\mathbf{s}_{n+1}=\mathbf{s}_{n+1}^{t r}-2 G \Delta \gamma \mathbf{n}_{n+1} .
$$


From the definition of the relative stress, $\boldsymbol{\beta}_{n+1}=\mathbf{s}_{n+1}-\mathbf{q}_{n+1}$, one has

$$
\begin{aligned}
\boldsymbol{\beta}_{n+1} & =\mathbf{s}_{n+1}^{t r}-2 G \Delta \gamma \mathbf{n}_{n+1}-\left(\mathbf{q}_{n}+\sqrt{\frac{2}{3}} \Delta H_{k}(\alpha) \mathbf{n}_{n+1}\right) \\
& =\boldsymbol{\beta}_{n+1}^{t r}-\left(2 G \Delta \gamma+\sqrt{\frac{2}{3}} \Delta H_{k}\left(\alpha_{n+1}\right)\right) \mathbf{n}_{n+1} .
\end{aligned}
$$

Notice the denotation of $\mathbf{n}_{n+1}$, the following relation is obtained

$$
\boldsymbol{\beta}_{n+1}\left(1+\frac{2 G \Delta \gamma+\sqrt{\frac{2}{3}} \Delta H_{k}\left(\alpha_{n+1}\right)}{\left\|\boldsymbol{\beta}_{n+1}\right\|}\right)=\boldsymbol{\beta}_{n+1}^{t r} .
$$

Since the term in the parentheses is a scalar, it can be inferred that $\boldsymbol{\beta}_{n+1}$ and $\boldsymbol{\beta}_{n+1}^{t r}$ point the same direction,

$$
\frac{\boldsymbol{\beta}_{n+1}}{\left\|\boldsymbol{\beta}_{n+1}\right\|}=\frac{\boldsymbol{\beta}_{n+1}^{t r}}{\left\|\boldsymbol{\beta}_{n+1}^{t r}\right\|} .
$$

Substituting above relation into Eq. (28), one obtains

$$
\left\|\boldsymbol{\beta}_{n+1}\right\|+2 G \Delta \gamma+\sqrt{\frac{2}{3}} \Delta H_{k}\left(\alpha_{n+1}\right)=\left\|\boldsymbol{\beta}_{n+1}^{t r}\right\| .
$$

Yield condition gives

$$
\begin{aligned}
f_{n+1} & =\left\|\boldsymbol{\beta}_{n+1}\right\|-\sqrt{\frac{2}{3}} H_{i}\left(\alpha_{n+1}\right) \\
& =\left\|\boldsymbol{\beta}_{n+1}^{t r}\right\|-2 G \Delta \gamma-\sqrt{\frac{2}{3}} \Delta H_{k}\left(\alpha_{n+1}\right)-\sqrt{\frac{2}{3}} H_{i}\left(\alpha_{n+1}\right) \\
& =0 .
\end{aligned}
$$

This equation is in general a non-linear equation and can be solved iteratively using Newton-Raphson scheme for $\Delta \gamma$. Once $\Delta \gamma$ is obtained, the values of step $n+1$ can be updated as follows

$$
\begin{aligned}
\varepsilon_{n+1}^{p} & =\varepsilon_{n}^{p}+\Delta \gamma \frac{\boldsymbol{\beta}_{n+1}^{t r}}{\left\|\boldsymbol{\beta}_{n+1}^{t r}\right\|}, \\
\boldsymbol{\alpha}_{n+1} & =\boldsymbol{\alpha}_{n}+\Delta \gamma \sqrt{\frac{2}{3}} \\
\mathbf{q}_{n+1} & =\mathbf{q}_{n}+\frac{2}{3} H_{k}^{\prime}(\alpha) \Delta \gamma \frac{\boldsymbol{\beta}_{n+1}^{t r}}{\left\|\boldsymbol{\beta}_{n+1}^{t r}\right\|} .
\end{aligned}
$$


The consistent elastoplastic material tensor is obtained by

$$
\begin{aligned}
\mathbb{C}^{e p} & =\frac{d \boldsymbol{\sigma}_{n+1}}{d \varepsilon_{n+1}} \\
& =\frac{d \boldsymbol{\sigma}_{n+1}^{t r}}{d \varepsilon_{n+1}}-2 G \frac{d\left(\Delta \gamma \mathbf{n}_{n+1}\right)}{d \varepsilon_{n+1}} \\
& =\mathbb{C}-2 G \frac{d \Delta \gamma}{d \varepsilon_{n+1}} \mathbf{n}_{n+1}-2 G \Delta \gamma \frac{d \mathbf{n}_{n+1}}{d \varepsilon_{n+1}} .
\end{aligned}
$$

From the yield condition

$$
f_{n+1}=\left\|\beta_{n+1}^{t r}\right\|-2 G \Delta \gamma-\sqrt{\frac{2}{3}} \Delta H_{k}\left(\alpha_{n+1}\right)-\sqrt{\frac{2}{3}} H_{i}\left(\alpha_{n+1}\right)=0,
$$

the derivative is taken as

$$
\frac{d f_{n+1}}{d \varepsilon_{n+1}}=\frac{d\left\|\beta_{n+1}^{t r}\right\|}{d \varepsilon_{n+1}}-2 G \frac{d \Delta \gamma}{d \varepsilon_{n+1}}-\sqrt{\frac{2}{3}} \frac{d \Delta H_{k}\left(\alpha_{n+1}\right)}{d \varepsilon_{n+1}}-\sqrt{\frac{2}{3}} \frac{d H_{i}\left(\alpha_{n+1}\right)}{d \varepsilon_{n+1}},
$$

in which the first term

$$
\begin{aligned}
\frac{d\left\|\boldsymbol{\beta}_{n+1}^{t r}\right\|}{d \varepsilon_{n+1}} & =\frac{d\left\|\boldsymbol{\beta}_{n+1}^{t r}\right\|}{d \boldsymbol{\beta}_{n+1}^{t r}} \frac{d \boldsymbol{\beta}_{n+1}^{t r}}{d \mathbf{s}_{n+1}^{t r}} \frac{d \mathbf{s}_{n+1}^{t r}}{d \varepsilon_{n+1}} \\
& =2 G \mathbf{n}_{n+1} \mathbf{I}^{d e v} \\
& =2 G \mathbf{n}_{n+1},
\end{aligned}
$$

the third term

$$
\begin{aligned}
\Delta H_{k}\left(\alpha_{n+1}\right) & =H_{k}\left(\alpha_{n+1}\right)-H_{k}\left(\alpha_{n}\right), \\
\sqrt{\frac{2}{3} \frac{d \Delta H_{k}\left(\alpha_{n+1}\right)}{d \varepsilon_{n+1}}} & =\frac{d H_{k}\left(\alpha_{n+1}\right)}{d \varepsilon_{n+1}} \\
& =\sqrt{\frac{2}{3}} \frac{d H_{k}\left(\alpha_{n+1}\right)}{d \alpha_{n+1}} \frac{d \alpha_{n+1}}{d \Delta \gamma} \frac{d \Delta \gamma}{d \varepsilon_{n+1}} \\
& =\frac{2}{3} H_{k}^{\prime}\left(\alpha_{n+1}\right) \frac{d \Delta \gamma}{d \varepsilon_{n+1}}
\end{aligned}
$$

and the fourth term

$$
\begin{aligned}
\sqrt{\frac{2}{3}} \frac{d \Delta H_{i}\left(\alpha_{n+1}\right)}{d \varepsilon_{n+1}} & =\frac{d H_{i}\left(\alpha_{n+1}\right)}{d \varepsilon_{n+1}} \\
& =\sqrt{\frac{2}{3}} \frac{d H_{i}\left(\alpha_{n+1}\right)}{d \alpha_{n+1}} \frac{d \alpha_{n+1}}{d \Delta \gamma} \frac{d \Delta \gamma}{d \varepsilon_{n+1}} \\
& =\frac{2}{3} H_{i}^{\prime}\left(\alpha_{n+1}\right) \frac{d \Delta \gamma}{d \varepsilon_{n+1}} .
\end{aligned}
$$


Since $\frac{d f_{n+1}}{d \varepsilon_{n+1}}=0$, the derivative $\frac{d \Delta \gamma}{d \varepsilon_{n+1}}$ is calculated as

$$
\begin{gathered}
\frac{d \Delta \gamma}{d \varepsilon_{n+1}}=\frac{2 G}{2 G+H_{i, n+1}^{\prime}+H_{k, n+1}^{\prime}} \mathbf{n}_{n+1}=\frac{1}{1+\frac{H_{i, n+1}^{\prime}+H_{k, n+1}^{\prime}}{3 G}} \mathbf{n}_{n+1}, \\
\frac{d \mathbf{n}_{n+1}}{d \varepsilon_{n+1}}=\frac{d \frac{\boldsymbol{\beta}_{n+1}^{t r}}{\left\|\boldsymbol{\beta}_{n+1}^{t r}\right\|}}{d \boldsymbol{\beta}_{n+1}^{t r}} \frac{d \boldsymbol{\beta}_{n+1}^{t r}}{d \varepsilon_{n+1}} \\
=\frac{1}{\left\|\boldsymbol{\beta}_{n+1}^{t r}\right\|}\left(\mathbf{I}-\mathbf{n}_{n+1} \otimes \mathbf{n}_{n+1}\right) 2 G \mathbf{I}^{d e v} \\
=\frac{2 G}{\left\|\boldsymbol{\beta}_{n+1}^{t r}\right\|}\left(\mathbf{I}^{d e v}-\mathbf{n}_{n+1} \otimes \mathbf{n}_{n+1}\right) .
\end{gathered}
$$

Substituting Eqs. (41) and (42) into Eq. (35), the consistent elastoplastic material tensor becomes

$$
\mathbb{C}^{e p}=\mathbb{C}-2 G\left(\frac{1}{1+\frac{H_{i, n+1}^{\prime}+H_{k, n+1}^{\prime}}{3 G}}-\frac{2 G \Delta \gamma}{\left\|\boldsymbol{\beta}_{n+1}^{t r}\right\|}\right) \mathbf{n}_{n+1} \otimes \mathbf{n}_{n+1}-\frac{4 G^{2} \Delta \gamma}{\left\|\boldsymbol{\beta}_{n+1}^{t r}\right\|} \mathbf{I}^{d e v}
$$

\section{NUMERICAL EXAMPLES}

To demonstrate the efficiency of the method, a long metallic thick-walled tube subjected to internal pressure is considered. The length of the tube is enough large to the width in order to solve this problem under plane strain conditions. The internal and external radius are $100 \mathrm{~mm}$ and $200 \mathrm{~mm}$, respectively. Fig. 2 illustrates the geometry of this problem. The material properties are: Young's modulus: $E=210 \mathrm{GPa}$, Possion's ratio: $v=0.3$, Uniaxial yield stress: $\sigma_{y}=0.24 \mathrm{GPa}$. The von Mises yield criterion is assumed

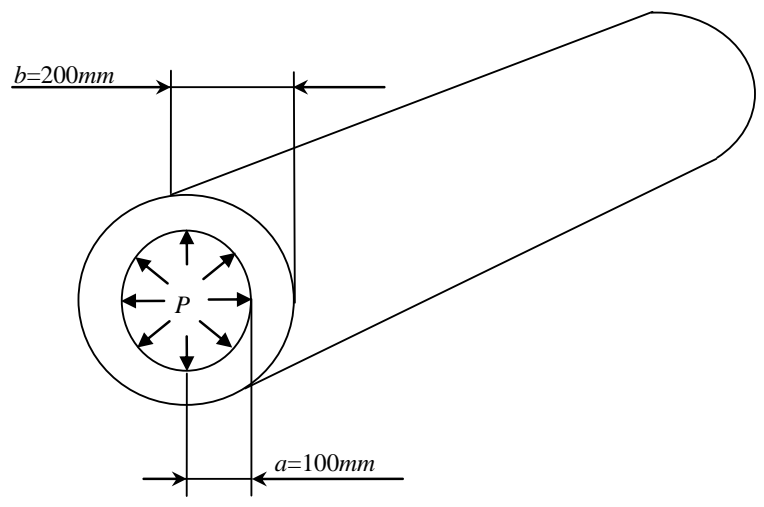

Fig. 2. Geometry and dimensions of internally pressurized tube

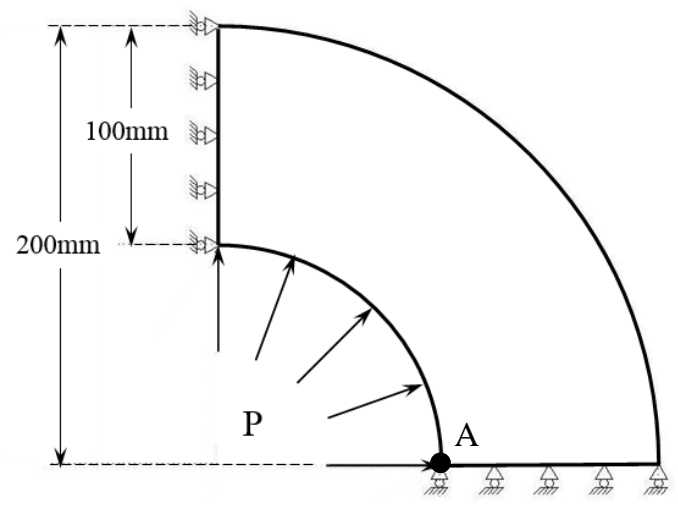

Fig. 3. A quarter model of internally pressurized tube 
bilinear hardening (tangent modulus $E_{t}=42 \mathrm{GPa}$ ). Due to its symmetry, the problem can be modeled by one quarter of the cross-section of the tube as shown in Fig. 3. The computation is performed using 20 load step increments in each load step time.

\subsection{A study of the convergence}

To check the accuracy and convergence, the internal pressure $\mathrm{P}=0.25 \mathrm{GPa}$ is considered. Data for meshes of a quarter tube are described in Appendix A and Fig. 4. We study the performance of the 2-D quadratic $(p=2)$, cubic $(p=3)$ and quartic $(p=4)$ NURBS basis function elements in comparison with quadratic finite elements derived from ANSYS Workbench software. As provided a reference solution for this problem, we use ANSYS solution with a very fine mesh of $500 \times 500$ quadratic elements $(1504002$ DOFs). The obtained $u_{r e f}$ and $\sigma_{r e f}$ values are $0.45123 \mathrm{~mm}$ and $0.42272 \mathrm{GPa}$, respectively. The relative errors are calculated by Eq. (44)

$$
\varepsilon_{A}=\left|\frac{u_{A}-u_{r e f}}{u_{r e f}}\right| \cdot 100[\%], \quad \varepsilon_{\sigma}=\left|\frac{\sigma_{A}-\sigma_{r e f}}{\sigma_{r e f}}\right| \cdot 100[\%],
$$

where $u_{r e f}$ and $\sigma_{r e f}$ are the reference solutions of $x$-displacement and von Mises equivalent stress, respectively. $u_{A}$ and $\sigma_{A}$ denote the computed value of $x$-displacement and von Mises equivalent stress at point $A$, respectively.

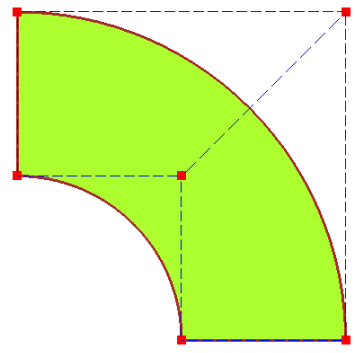

(a) Coarsest mesh

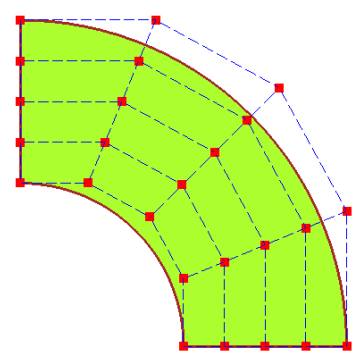

(b) A quartic element

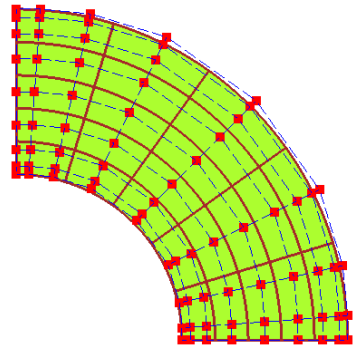

(c) $5 \times 5$ quartic elements

Fig. 4. The meshing of internally pressurized tube

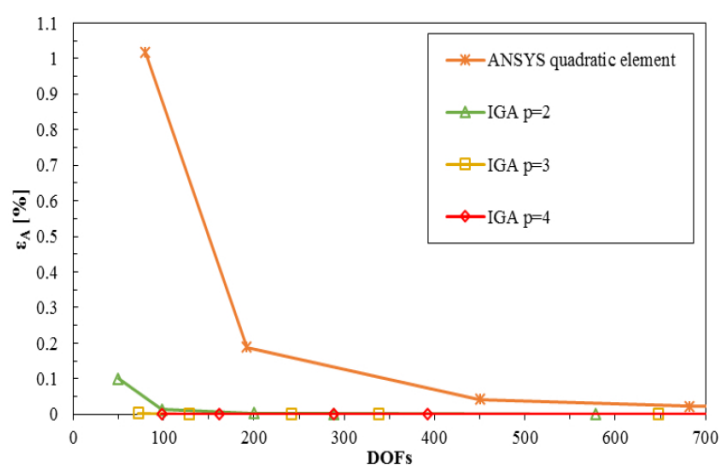

(a)

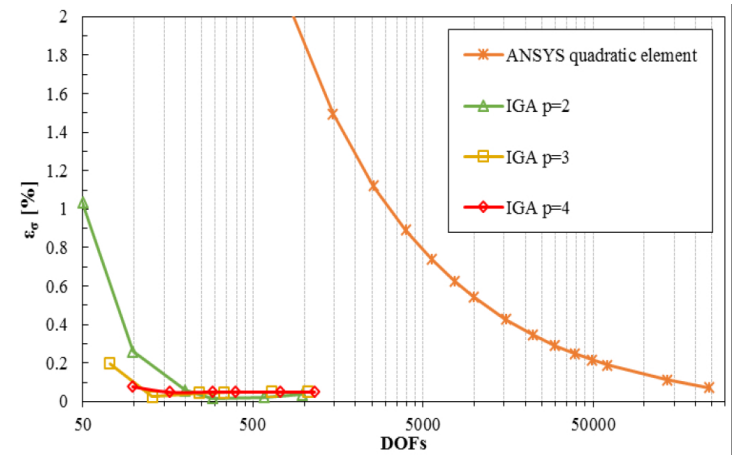

(b)

Fig. 5. The comparison of the relative errors of (a) the $x$-displacement and (b) the von Mises equivalent stress at point $A$ 
The relative errors of $x$-displacement and von Mises equivalent stress versus the total number of degree of freedoms (DOFs) at point A are displayed in Fig. 5(a) and Fig. 5(b), respectively. It is observed from Fig. 5(a) that the present results are almost identical (with the error $\varepsilon_{A}<0.1 \%$ ) to the reference solution of $x$-displacement for few DOFs $(5 \times 5$ quadratic NURBS elements or approximately 98 DOFs) while ANSYS solution requires a large number of degree of freedoms (approximately 450 DOFs). In the other hand, Fig. 5(b) shows the relative error of von Mises equivalent stress. We need a large number of degree of freedoms of ANSYS (approximately 241602 DOFs) to adapt (the error $\varepsilon_{\sigma}<0.1 \%$ ) the von Mises equivalent stress reference value while the IGA solution needs only 98 DOFs with meshing of $3 \times 3$ quartic NURBS elements. It is highly noted that the present method, that just uses a slighly coarse mesh of $3 \times 3$ quartic NURBS elements in both displacement and equivalent stress results, produces very accurate solution compared to the reference solution. For this reason, we recommend meshing of $5 \times 5$ quartic NURBS elements (Fig. 4(c)) as a good candidate for below problems. It shows clearly an enormous advantage of the present method. The distributions of total displacement, von Mises equivalent stress and plastic strain solutions of the long metallic thick-walled tube are illustrated in Fig. 6.

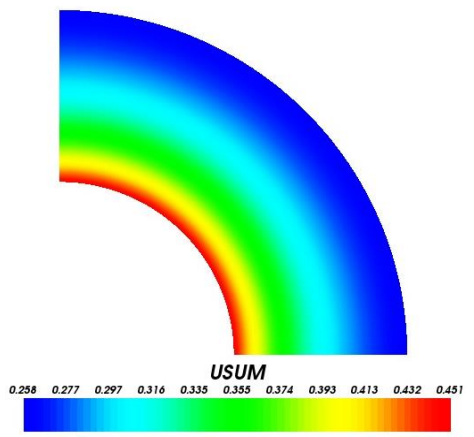

(a)

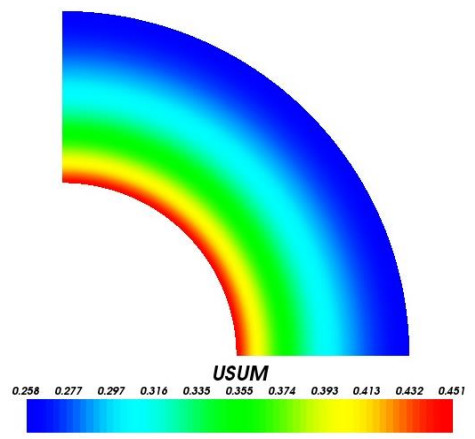

(b)

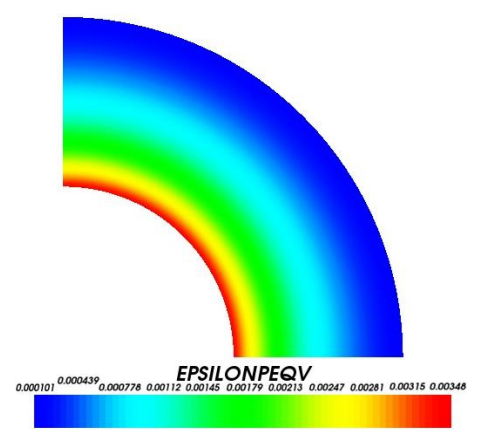

(c)

Fig. 6. The distributions of (a) the total displacement, (b) the von Mises equivalent stress and (c) the plastic strain solution

\subsection{A study of the hardening}

In this section, series of loading-unloading with the maximum loading are higher after each cycle, as shown in Fig. 7, is applied to investigate the "hardening" phenomenon. The pressure-displacement curve in Fig. 8 shows clearly the "hardening" behavior. At first, the internal pressure is gradually increased from 0 to $0.25 \mathrm{GPa}$ (Fig. 7), causing the material behavior changing from linear elasticity to plasticity (Fig. 8). Then the load is released. The part of the pressure-displacement curve corresponding to unloading is a straight line whose slope is the elastic modulus, showing the elastic recovery. The load is then increased. The interesting "hardening" is observed, as the pressure-displacement is a straight line, characteristic to elastic behavior, until the load reaches $0.25 \mathrm{GPa}$ and when 
the load is more than $0.25 \mathrm{GPa}$, the response displays plastic behavior. Noting that 0.25 GPa is the maximum load ever reached and it is higher than the yield stress, which is 0.24 GPa. This means that the material is "hardened" and now the yield stress is shifted to a higher level, equal to the maximum load ever applied. The loading is continued until the pressure reaches $0.275 \mathrm{GPa}$, then followed by unloading. Again, elastic recovery is observed. Then loading is applied again. Similarly, the material displays elastic behavior until the load reaches the maximum load ever reached, i.e. $0.275 \mathrm{GPa}$, and turns to plastic behavior after that level. When the pressure reaches $0.3 \mathrm{GPa}$, unloading is again applied and it is obviously that the response shows elastic recovery.

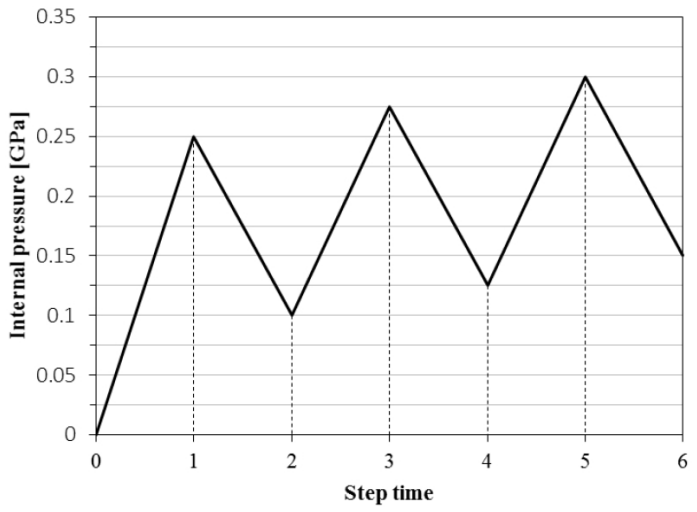

Fig. 7. Loading history of internal pressure

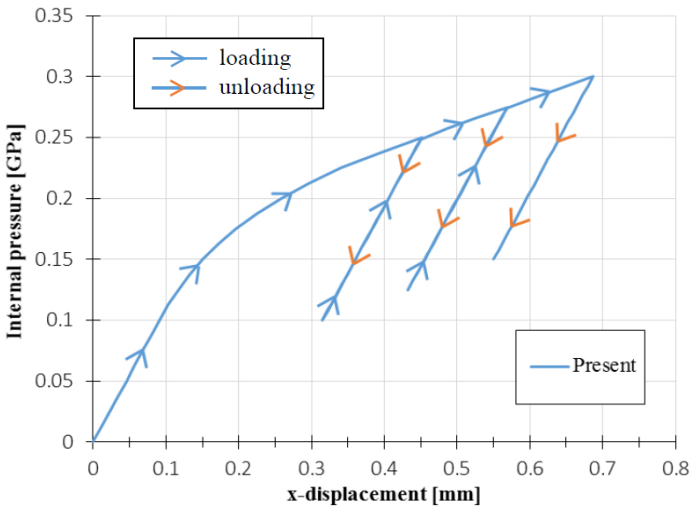

Fig. 8. The internal pressure versus $x$-displacement at point A to check hardening behavior. The arrows denote the direction of loadingunloading

This test shows that the present model is able to capture well the hardening behavior under tensile condition. However, in tensile test, it is not possible to distinguish whether the hardening is isotropic or kinematic.

\subsection{A study of the cycling loading}

Next, to study the von Mises model with linear isotropic hardening and kinematic hardening, series of cyclic tension-compression loading is applied, as illustrated in Fig. 9. The difference between isotropic hardening and kinematic hardening is the difference in material behavior under cyclic tension-compression loading, and it is visualized in Fig. 10. The results obtained by the present method are in good agreement with those derived from the commercial software ANSYS Workbench. The kinematic hardening model, shown in Fig. 10(b), is clearly distinguished from the isotropic hardening model, shown in Fig. 10(a), as Bauschinger effect is observed in the kinematic hardening model. Bauschinger effect implies that the yield strength of metal decreases as the direction of strain is changed, i.e. change from tension to compression and vice versa. For example, an increase in tensile yield strength leads to the reduction of compressive yield strength. The kinematic hardening model, with the assumption that the yield surface 
does not change in term of size but translates following the loading condition, is able to capture the Bauschinger effect.

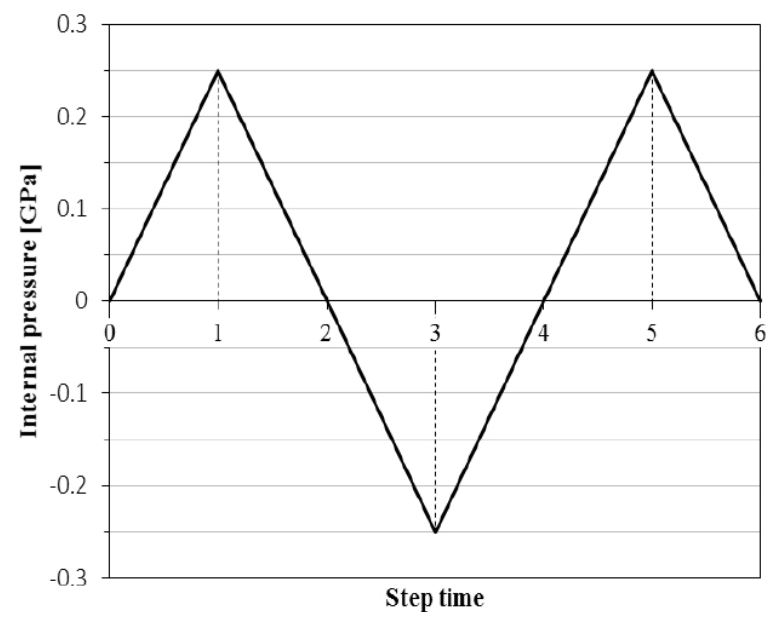

Fig. 9. Loading history of internal pressure

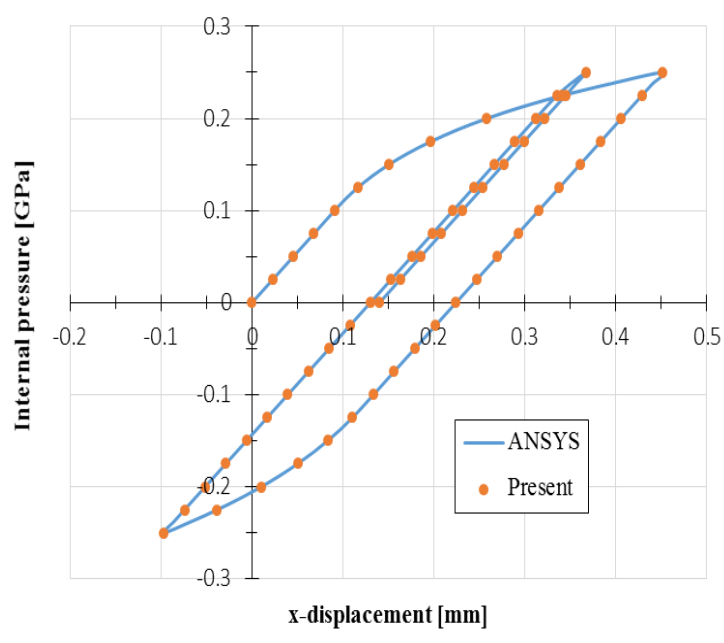

(a)

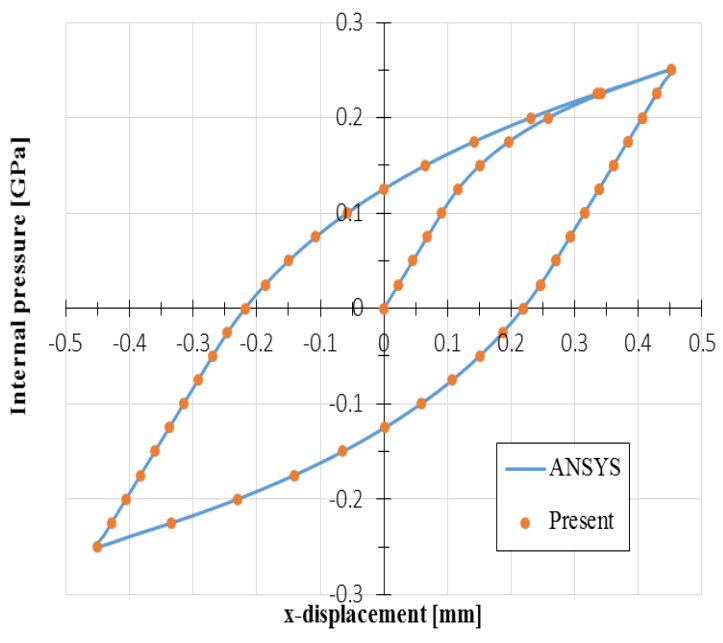

(b)

Fig. 10. The Internal pressure versus $x$-displacement at point $A$ (a) The kinematic hardening and (b) linear isotropic hardening

\subsection{A study of the spring-back phenomenon}

To study the spring back phenomenon, a metal pipe is considered. Spring-back occurs when a structure is loaded over the yield limit and then the load is released. Due to the irreversibility of the plastic deformation, the structure does not recover to the initial 


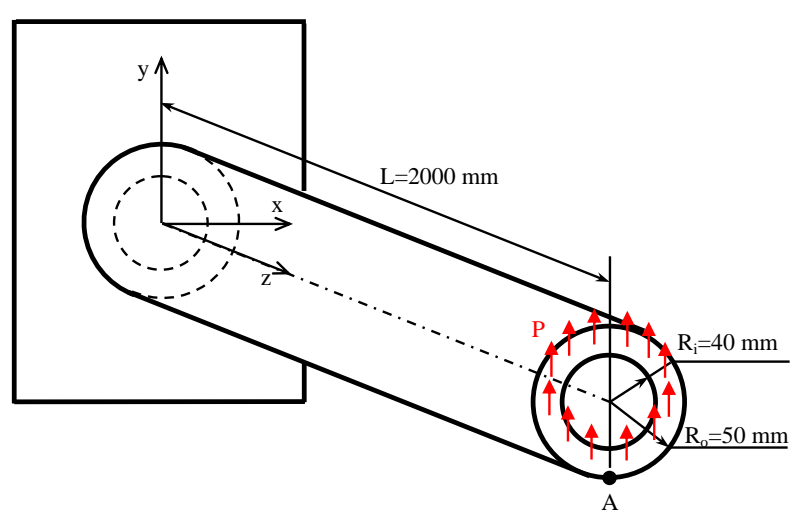

Fig. 11. Geometry of the pipe clamped on one end and loaded on the other end

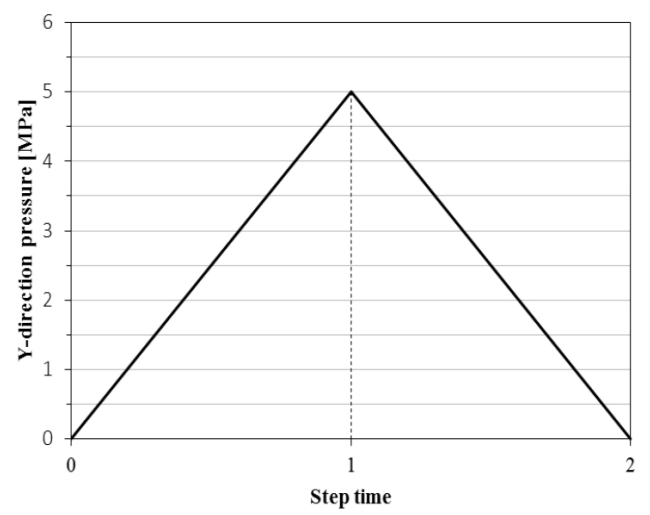

Fig. 12. Loading history of uniform $y$-direction pressure

configuration (before the load is applied), but only the elastic part of the deformation is recovered. In metal forming, in order to obtain the desired deformation, it is necessary to compensate the spring-back phenomenon, by applying a larger load, such that after the load is released, the shape is sprung back to the desired one. Here, only the springback phenomenon is presented. However, the algorithm that compensates the springback phenomenon is not within the scope of this study. In this example, the pipe is modeled in three-dimension (Fig. 11). One end of the pipe is clamped and the other end is subject to load. The internal radius, outer radius and length of the pipe are $40 \mathrm{~mm}, 50$ $\mathrm{mm}$ and $2000 \mathrm{~mm}$, respectively. A uniform pressure $\mathrm{P}$ is applied on the free-end of the pipe on $y$-direction with loading path shown in Fig. 12. It is assumed that the material experiencing von Mises yield criterion with linear hardening. The material properties are as follows: Young's modulus $E=207000 \mathrm{MPa}$, Possion's ratio: $v=0.3$, uniaxial yield stress: $\sigma_{y}=207 \mathrm{MPa}$, and tangent modulus $E_{t}=25900 \mathrm{MPa}$. Data for the coarsest mesh of the pipe is described in Appendix B, Fig. 13(a) and Fig. 13(c). It is observed from Fig. 5 that quartic NURBS gives better results in terms of accuracy. In this problem, the initial quartic mesh (Fig. 13(b)) is obtained by elevating the NURBS degree from the coarsest mesh. A mesh of 320 three-dimensional quartic NURBS elements is chosen to compute the problem, i.e. 10440 DOFs, as shown in Fig. 13(d).

The results of displacement are depicted in Fig. 14, showing clearly the shape of the pipe in three configurations: initially un-deformed shape, deformed shape at peak load, and the shape after load released totally. It is observed that the pipe does not recover to the un-deformed configuration. This reason is because the region near the clamped end of the pipe is already yielded, as shown by the distribution of plastic strain in Fig. 15.

The graph in Fig. 13 presents the displacement at point A (see Fig. 16) with respected to the pressure applied obtained by IGA, compared with that obtained by the software ANSYS. An excellent agreement is observed. As the pressure increases, deformation increases. Non-linearity occurs when part of the pipe experiences plastic yielding. When the load is released, the elastic part of the deformation is recovered. When the load is totally released, the pipe is still deformed, due to existence of plastic deformation. 


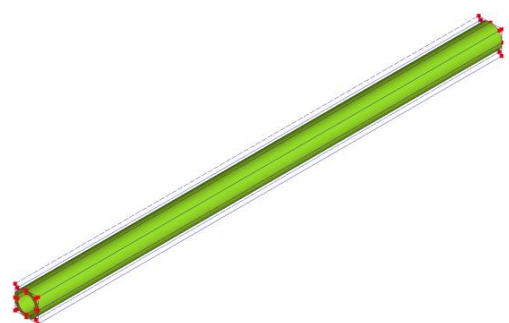

(a)

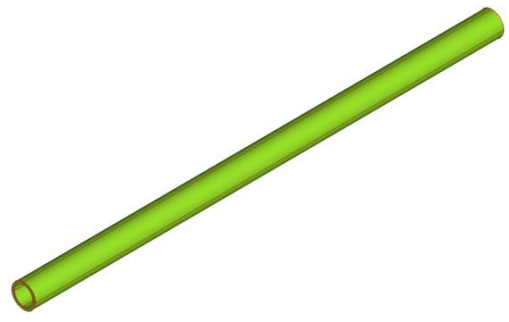

(c)

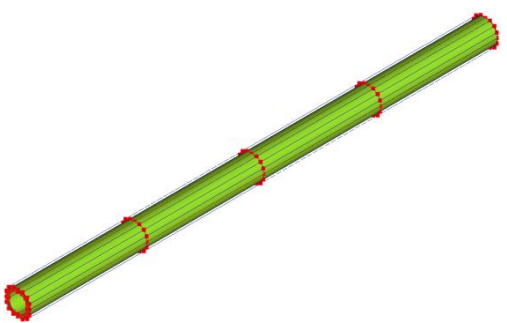

(b)

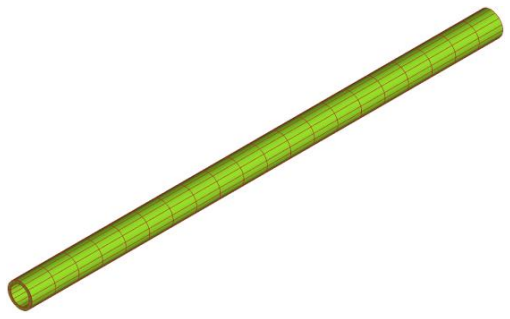

(d)

Fig. 13. The meshing of 3D pipeline: (a) coarsest mesh and control points;

(b) a 4 - quartic - element - mesh and control points; (c) coarsest mesh with 4 elements; and (d) computing mesh with 320 elements

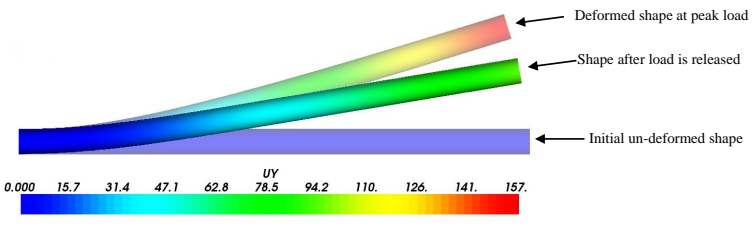

Fig. 14. Distribution of $y$-displacement in three time steps

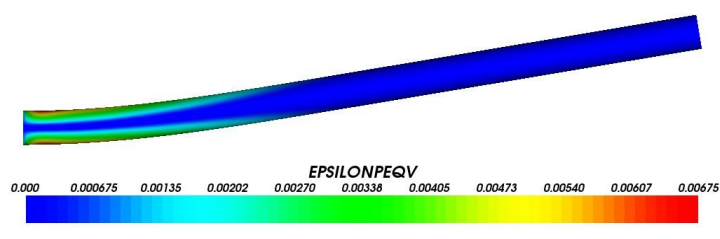

(a)

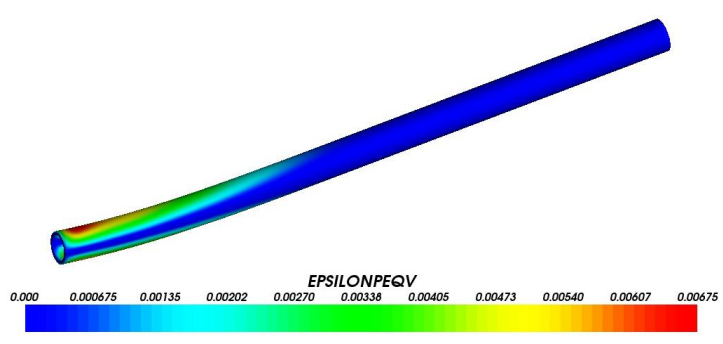

(b)

Fig. 15. The plastic strain of pipeline at end step: (a) front view and (b) 3D view 


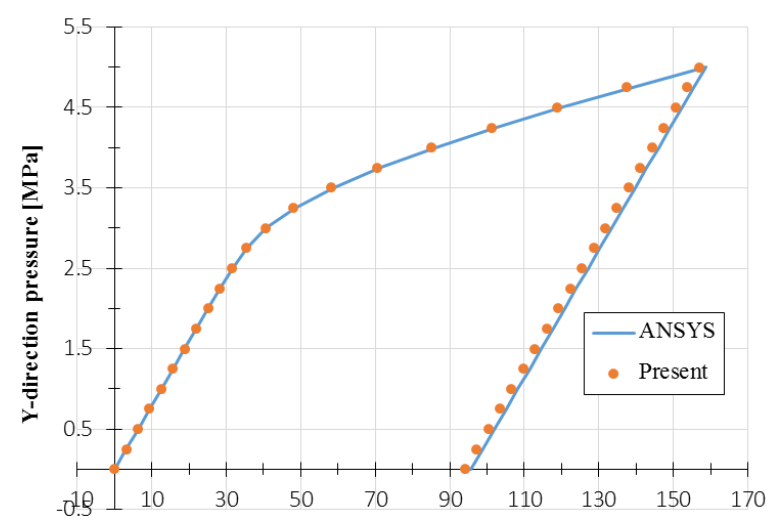

Fig. 16. The $y$-direction pressure versus $y$-displacement at point $\mathrm{A}[\mathrm{mm}]$

\section{CONCLUSIONS}

We presented an effectively computational tool based on an isogeometric finite element approach. Numerical validation is tested for a long metallic thick-walled tube with linear isotropic hardening and kinematic hardening of von Mises model. Higher-order NURBS basis functions were used for modeling geometry and obtaining approximate solutions. The method allows us to design exactly arbitrary geometry at a coarse mesh level and to achieve highly accurate solutions. We found that the quartic NURBS element is a great choice for providing an ultra-accurate solution with a low number of degrees of freedom. Its result is well competitive with other existing and ANSYS solutions. The method is also promising for analyzing other elastoplastic problems using return-mapping algorithm for von Mises yield criterion through NURBS basis functions.

\section{ACKNOWLEDGMENT}

This research is funded by Vietnam National Foundation for Science and Technology Development (NAFOSTED) under grant number 107.02-2014.24. The support is gratefully acknowledged.

\section{REFERENCES}

[1] J. C. Simo and T. J. R. Hughes. Computational inelasticity, Vol. 7. Springer Science \& Business Media, (2006). doi:10.1007/b98904.

[2] R. D. Krieg and D. B. Krieg. Accuracies of numerical solution methods for the elasticperfectly plastic model. Journal of Pressure Vessel Technology, 99, (4), (1977), pp. 510-515. doi:10.1115/1.3454568.

[3] Z. Wei, D. Perić, and D. Owen. Consistent linearization for the exact stress update of PrandtlReuss non-hardening elastoplastic models. International Journal for Numerical Methods in En-gineering, 39, (7), (1996), pp. 1219-1235. doi:10.1002/(sici)1097-0207(19960415)39:7 $<1219::$ aid-nme901>3.0.co;2-7. 
[4] L. Szabó. A semi-analytical integration method for J2 flow theory of plasticity with linear isotropic hardening. Computer Methods in Applied Mechanics and Engineering, 198, (27), (2009), pp. 2151-2166. doi:10.1016/j.cma.2009.02.007.

[5] M. Ristinmaa and J. Tryding. Exact integration of constitutive equations in elasto-plasticity. International Journal for Numerical Methods in Engineering, 36, (15), (1993), pp. 2525-2544. doi:10.1002/nme.1620361503.

[6] E. A. de Souza Neto, D. Peric, and D. R. J. Owen. Computational methods for plasticity: theory and applications. John Wiley \& Sons, (2011).

[7] M. Theofilos-Ioannis. Elastoplastic constitutive models in finite element analysis. National Technical University Athens, (2012).

[8] J. S. Chen, C. M. O. L. Roque, C. Pan, and S. T. Button. Analysis of metal forming process based on meshless method. Journal of Materials Processing Technology, 80, (1998), pp. 642-646. doi:10.1016/s0924-0136(98)00171-x.

[9] V. Petersson. An implementation of mesh free methods for mechanical problems at large strains. Master's thesis, Division of Solid Mechanics, Lund University, (2007).

[10] M. N. Nguyen and V. L. Ha. Application of meshless method for plastic deformation in 2D. Ho Chi Minh City University of Technology, (2008).

[11] M. A. Elgammi. A boundary/finite element coupled method for elasto-plastic deformation analysis. Atilim University, (2010).

[12] D. N. Cathie. On the implementation of elasto-plastic boundary element analysis. Applied Mathematical Modelling, 5, (1), (1981), pp. 39-44. doi:10.1016/0307-904x(81)90057-3.

[13] J. C. Simo and R. L. Taylor. Consistent tangent operators for rate-independent elastoplasticity. Computer Methods in Applied Mechanics and Engineering, 48, (1), (1985), pp. 101-118. doi:10.1016/s0045-7825(85)90054-4.

[14] T. J. R. Hughes, J. A. Cottrell, and Y. Bazilevs. Isogeometric analysis: CAD, finite elements, NURBS, exact geometry and mesh refinement. Computer Methods in Applied Mechanics and Engineering, 194, (39), (2005), pp. 4135-4195. doi:10.1016/j.cma.2004.10.008.

[15] J. A. Cottrell, T. J. R. Hughes, and Y. Bazilevs. Isogeometric analysis: toward integration of CAD and FEA. John Wiley \& Sons, (2009).

[16] J. A. Cottrell, A. Reali, Y. Bazilevs, and T. J. R. Hughes. Isogeometric analysis of structural vibrations. Computer Methods in Applied Mechanics and Engineering, 195, (41-43), (2006), pp. 5257-5296. doi:10.1016/j.cma.2005.09.027.

[17] L. B. da Veiga, A. Buffa, C. Lovadina, M. Martinelli, and G. Sangalli. An isogeometric method for the Reissner-Mindlin plate bending problem. Computer Methods in Applied Mechanics and Engineering, 209, (2012), pp. 45-53. doi:10.1016/j.cma.2011.10.009.

[18] N. Valizadeh, T. Q. Bui, V. T. Vu, H. T. Thai, and M. N. Nguyen. Isogeometric simulation for buckling, free and forced vibration of orthotropic plates. International Journal of Applied Mechanics, 5, (02), (2013). doi:10.1142/s1758825113500178.

[19] J. Kiendl, K. U. Bletzinger, J. Linhard, and R. Wüchner. Isogeometric shell analysis with Kirchhoff-Love elements. Computer Methods in Applied Mechanics and Engineering, 198, (49), (2009), pp. 3902-3914. doi:10.1016/j.cma.2009.08.013.

[20] D. J. Benson, Y. Bazilevs, M. C. Hsu, and T. J. R. Hughes. Isogeometric shell analysis: the Reissner-Mindlin shell. Computer Methods in Applied Mechanics and Engineering, 199, (5), (2010), pp. 276-289. doi:10.1016/j.cma.2009.05.011. 
[21] T. Elguedj, Y. Bazilevs, V. M. Calo, and T. J. R. Hughes. $\bar{B}$ and $\bar{F}$ projection methods for nearly incompressible linear and non-linear elasticity and plasticity using higher-order NURBS elements. Computer Methods in Applied Mechanics and Engineering, 197, (33-40), (2008), pp. 2732 2762. doi:10.1016/j.cma.2008.01.012.

[22] A. Buffa, G. Sangalli, and R. Vázquez. Isogeometric analysis in electromagnetics: B-splines approximation. Computer Methods in Applied Mechanics and Engineering, 199, (17), (2010), pp. 1143-1152. doi:10.1016/j.cma.2009.12.002.

[23] H. Gómez, V. M. Calo, Y. Bazilevs, and T. J. R. Hughes. Isogeometric analysis of the CahnHilliard phase-field model. Computer Methods in Applied Mechanics and Engineering, 197, (49), (2008), pp. 4333-4352. doi:10.1016/j.cma.2008.05.003.

[24] M. J. Borden, C. V. Verhoosel, M. A. Scott, T. J. R. Hughes, and C. M. Landis. A phase-field description of dynamic brittle fracture. Computer Methods in Applied Mechanics and Engineering, 217, (2012), pp. 77-95. doi:10.1016/j.cma.2012.01.008.

[25] L. Piegl and W. Tiller. The NURBS book. Springer Berlin Heidelberg, (1995). doi:10.1007/9783-642-97385-7.

\section{APPENDIX A: DATA FOR A THICK-WALLED TUBE MODEL}

The 2-D geometry for a thick-walled tube model in Section 4 uses a bivariate NURBS basis function. There are two parametric directions, $\xi$ and $\eta$, are corresponded to the circumferential and radial directions, respectively. The corresponding polynomial degrees and knot vectors are given in Tab. 1 and the control points are given in Tab. 2.

Table 1. Polynomial degrees and knot vectors for a thick-walled tube model

\begin{tabular}{|c|c|c|}
\hline Direction & Degree & Knot vector \\
\hline$\xi$ & $p=2$ & $\boldsymbol{\Xi}=\{0,0,0,1,1,1\}$ \\
\hline$\eta$ & $q=1$ & $\boldsymbol{\Xi}=\{0,0,1,1\}$ \\
\hline
\end{tabular}

Table 2. Control points for a thick-walled tube model

\begin{tabular}{|c|c|c|c|c|}
\hline$i$ & $\mathbf{P}_{i, 1}$ & $\mathbf{P}_{i, 2}$ & $w_{i, 1}$ & $w_{i, 2}$ \\
\hline 1 & $(100,0)$ & $(200,0)$ & 1 & 1 \\
\hline 2 & $(100,100)$ & $(200,200)$ & $\sqrt{2} / 2$ & $\sqrt{2} / 2$ \\
\hline 3 & $(0,100)$ & $(0,100)$ & 1 & 1 \\
\hline
\end{tabular}

\section{APPENDIX B: DATA FOR A 3D PIPELINE MODEL}

The 3-D geometry for a pipeline model in Section 4 uses a trivariate NURBS basis function. There are three parametric directions, $\xi, \eta$, and $\zeta$ are corresponded to the radial, circumferential and longitudinal directions, respectively. The corresponding polynomial degrees and knot vectors are given in Tab. 3 and the control points are given in Tab. 4 . 
Table 3. Polynomial degrees and knot vectors for a pipeline model

\begin{tabular}{|c|c|c|}
\hline Direction & Degree & Knot vector \\
\hline$\zeta$ & $p=1$ & $\boldsymbol{\Xi}=\{0,0,1,1\}$ \\
\hline$\eta$ & $q=2$ & $\boldsymbol{\Xi}=\{0,0,0,0.25,0.25,0.5,0.5,0.75,0.75,1,1,1\}$ \\
\hline$\zeta$ & $r=1$ & $\mathbf{\Xi}=\{0,0,1,1\}$ \\
\hline
\end{tabular}

Table 4. Control points for a pipeline model

\begin{tabular}{|c|c|c|c|c|c|}
\hline$j$ & $k$ & $\mathbf{P}_{1, j, k}$ & $\mathbf{P}_{2, j, k}$ & $w_{i, 1}$ & $w_{i, 2}$ \\
\hline 1 & 1 & $(40,0,0)$ & $(50,0,0)$ & 1 & 1 \\
\hline 2 & 1 & $(40,40,0)$ & $(50,50,0)$ & $\sqrt{2} / 2$ & $\sqrt{2} / 2$ \\
\hline 3 & 1 & $(0,40,0)$ & $(0,50,0)$ & 1 & 1 \\
\hline 4 & 1 & $(-40,40,0)$ & $(-50,50,0)$ & $\sqrt{2} / 2$ & $\sqrt{2} / 2$ \\
\hline 5 & 1 & $(-40,0,0)$ & $(-50,0,0)$ & 1 & 1 \\
\hline 6 & 1 & $(-40,-40,0)$ & $(-50,-50,0)$ & $\sqrt{2} / 2$ & $\sqrt{2} / 2$ \\
\hline 7 & 1 & $(0,-40,0)$ & $(0,-50,0)$ & 1 & 1 \\
\hline 8 & 1 & $(40,-40,0)$ & $(50,-50,0)$ & $\sqrt{2} / 2$ & $\sqrt{2} / 2$ \\
\hline 9 & 1 & $(40,0,0)$ & $(50,0,0)$ & 1 & 1 \\
\hline 1 & 2 & $(40,0,2000)$ & $(50,0,2000)$ & 1 & 1 \\
\hline 2 & 2 & $(40,40,2000)$ & $(50,50,2000)$ & $\sqrt{2} / 2$ & $\sqrt{2} / 2$ \\
\hline 3 & 2 & $(0,40,2000)$ & $(0,50,2000)$ & 1 & 1 \\
\hline 4 & 2 & $(-40,40,2000)$ & $(-50,50,2000)$ & $\sqrt{2} / 2$ & $\sqrt{2} / 2$ \\
\hline 5 & 2 & $(-40,0,2000)$ & $(-50,0,2000)$ & 1 & 1 \\
\hline 6 & 2 & $(-40,-40,2000)$ & $(-50,-50,2000)$ & $\sqrt{2} / 2$ & $\sqrt{2} / 2$ \\
\hline 7 & 2 & $(0,-40,2000)$ & $(0,-50,2000)$ & 1 & 1 \\
\hline 8 & 2 & $(40,-40,2000)$ & $(50,-50,2000)$ & $\sqrt{2} / 2$ & $\sqrt{2} / 2$ \\
\hline 9 & 2 & $(40,0,2000)$ & $(50,0,2000)$ & 1 & 1 \\
\hline & & & & & \\
\hline
\end{tabular}

